\title{
Short-Term Scheduling of a Polymer Compounding Plant
}

\author{
P. Castro ${ }^{\dagger}$, A. P. F. D. Barbosa-Póvoa ${ }^{* \dagger}$ and H. Matos ${ }^{\dagger}$ \\ ${ }^{\dagger}$ Departamento de Engenharia Química and ${ }^{\star}$ Centro de Estudos de Gestão \\ Instituto Superior Técnico, 1049-001, Lisboa, Portugal
}

\begin{abstract}
This paper addresses the optimal short-term scheduling of a three parallel production line polymer compounding plant, whose equipments require cleaning between product changeovers. A very effective user-friendly software tool was developed, which consists of a general scheduling model coupled with the capabilities of Microsoft Excel for data handling and analysis. The scheduling model is based on a Resource Task Network discrete time formulation and leads to Mixed Integer Linear Programming problems. As outputs the user can access the optimal schedules for a number of different objectives.
\end{abstract}

\section{Introduction}

The short-term schedule of a multiproduct plant is to be periodically made to satisfy a given set of production orders for different products within a fixed time horizon. The orders may come directly from customers or be generated to meet inventory requirements. In the multiproduct plant considered (a two-stage, three parallel line facility), the list of products to be manufactured changes every day. Hence, a cyclic production policy is inadequate to follow market demands and a non-regular production pattern is adopted. The scheduling of a given set of product orders involves important decisions regarding: $i$ ) the assignment of products to a given production line and $i$ ) product sequencing. The aim is to minimise changeover times and meet due dates. To help with this decision making process and to improve customer satisfaction an efficient scheduling tool was developed.

\section{Problem Analysis}

The process can be viewed as consisting of two limiting stages: $i$ ) mixing and $i i)$ extruding. Three batch mixers (CPE-049, CPE-055 and CPE-075) are suitable for the first stage while three semi-continuous extruders (CPE-001, CPE-002 and CPE-003) can handle the second stage. Each mixer has its own maximum and minimum capacities. Whenever the amount to be produced exceeds the maximum capacity of the mixer more than one batch is required. The extruding task can start as soon as the first mixing batch is completed.

\footnotetext{
* Corresponding author. Tel: +351-218419014. Fax: +351-218417638. E-mail: apovoa@ist.utl.pt
} 
The plant is capable of producing several products resulting from the incorporation and dispersion of different colours into polymers, usually low-density polyethylene. The number of colours that can be produced at the plant exceeds one thousand. These can be grouped into 15 different families (set $C$ ) according to their main tint, ranging from white to black. Every time a product is changed, the mixers and the extruders require cleaning. Changeover times are dependent on three entities: $i$ ) the equipment handling the product (an element of set $E$ ); $i$ ) the colour of the product that has been processed and iii) the colour of the product that is going to be processed immediately after. For a given equipment there are at most three distinct cleaning times for every colour tone. Also, cleaning from a colour tone to a different one lasts the same time regardless of the final colour tone. These two characteristics clearly suggest that, in certain situations, two or more colour tones can be grouped into a new family.

To reduce the number of colour tones to consider in each equipment and consequently the number of cleaning tasks required to model the process, a colourgrouping algorithm was developed. The algorithm identifies functional equivalences among subsets of the available resources, thereby allowing a more aggregate treatment of such resources (Dimitriadis et al.,1998). As a result, the RTN representation of the process becomes simpler and the size of the resulting formulation becomes smaller.

\section{Mathematical Model}

The general Resource Task Network (RTN) representation (Pantelides, 1994) is used to model the scheduling problem. The mathematical formulation is based on a discrete representation of time, where the horizon is divided into a number of intervals of equal and fixed duration, and gives rise to a MILP problem. All system events are forced to coincide with one of the interval boundaries.

\subsection{RTN Process Representation}

The RTN representation regards all processes as bipartite graphs comprising two types of nodes: resources and tasks. Each task is an operation that transforms a certain set of resources into another set. On the other hand, the relevant resources are classified into two different types: $i$ ) resources representing the relevant material states, set $R$; $i$ ) resources representing the two possible equipment states, set $S$.

The adopted superstructure, where the three production lines are clearly identified, is given in Figure 1. For each product, five different resources are required ( $R 1$ represents the raw materials, $R 2$ through $R 4$ represent the intermediate mixing states, one for each line, and $R 5$ the final product. Clean $(S 1)$ and dirty $(S 2)$ states are also considered. Besides the equipment index, these states need to be referred to a certain colour tone, omitted for simplicity. In Figure 1 it is assumed that the total amount of the mixed intermediate is produced at the end of the first mixing batch. These two situations are distinguished in the RTN: arrows denoting production of resources at the end of the first batch are connected to the block in an intermediate position while those denoting production at the end of the last batch are connected further to the right. Although the extruders operate semi-continuously, the extruding tasks are modelled as batch: the mixed product $(R 2, R 3$ or $R 4)$ is totally consumed at the beginning of the task, while the final product is totally produced at its end. 


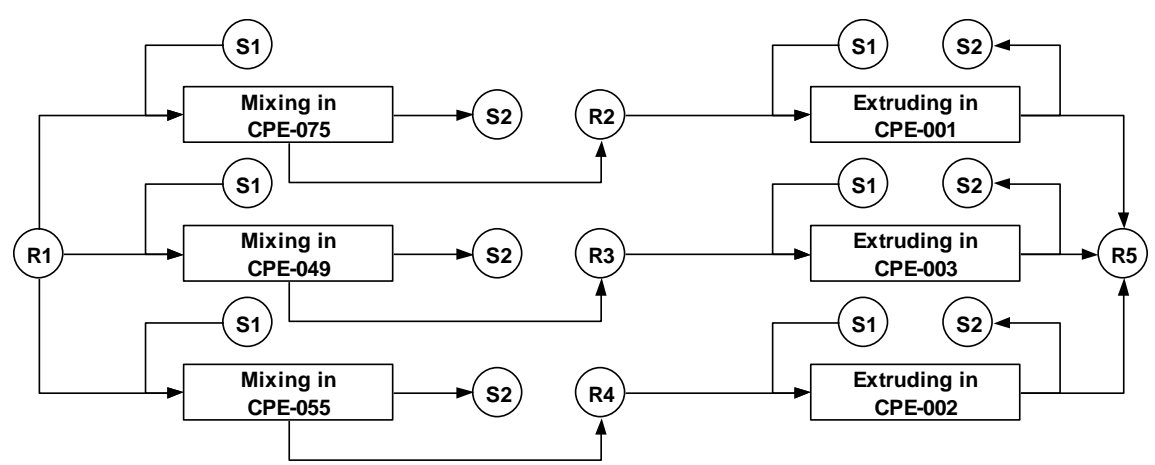

Figure 1-RTN process representation for each product. Part 1: processing tasks.

Between processing tasks the equipments require cleaning. Each cleaning task consumes a dirty state $(S 2)$ at its beginning and produces a clean state $(S 1)$ at its end.

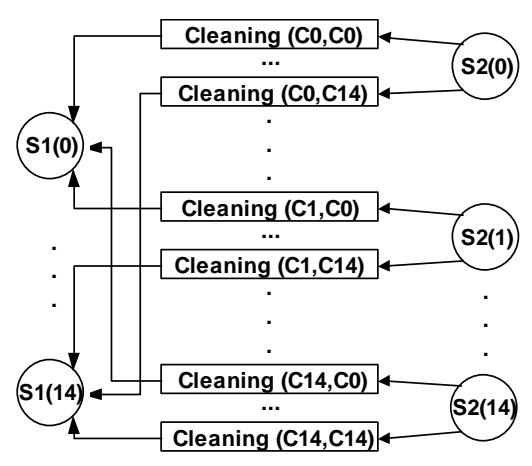

Figure 2- RTN for each equipment. Part 2: cleaning tasks.

However, it is not enough to refer to a piece of equipment as clean. It is also necessary to state which colour it has been cleaned for. As the process superstructure must include all possible colour combinations $(15 \times 15)$ for each equipment, its representation is quite complex (see Figure 2) The first cleaning task $(C 0, C 0)$ consumes the white dirty state $S 2(0)$ and produces the white clean state, $S 1(0)$. As the cleaning times vary between equipments, even for the same two colours, the number of cleaning tasks to consider is usually not the same in different equipments (a certain colour group will include more or less colours, see section 2).

\subsection{Mathematical Formulation}

The short-term scheduling of the plant is achieved by the following constraints.

Excess resource balances: the excess amount of a resource at a given time interval $t$ is equal to that at the previous time interval adjusted by the amount consumed by all tasks starting at the beginning of $t$ and by the amount produced by all tasks starting at a previous time interval and ending at $t$.

$$
\begin{aligned}
& R_{r, p, t}=R_{r, p, t-1}+\sum_{e \in E} \sum_{\theta=0}^{\tau_{e, p}^{P-1 B}} \mu_{e, r, p, \theta}^{P} N_{e, p, t-\theta}^{P} \forall r \in R, p \in P, t \in T \\
& S_{e, s, c, t}=S_{e, s, c, t-1}+\sum_{p \in P} \sum_{\theta=0}^{\tau_{e, p}^{P}} v_{e, p, s, c, \theta}^{P} N_{e, p, t-\theta}^{P}+\left.\sum_{c^{\prime} \in C} \sum_{\theta=0}^{\tau_{e, c, c^{\prime}}} v_{e, c, s, c^{\prime}, \theta}^{L}\right|_{s=2 \wedge \theta=0} N_{e, c, c^{\prime}, t-\theta}^{L}+\left.\gamma_{e, s, c, t}\right|_{s=2} \\
& -\left.N B_{e, c, t}\right|_{s=2 \wedge e>3}+\left.\sum_{c^{\prime} \in C} \sum_{\theta=0}^{\tau_{e, c^{\prime}, c}^{L}} \bar{v}_{e, c^{\prime}, c, s, \theta}^{L}\right|_{s=1 \wedge \theta=\tau_{e, c, c^{\prime}}^{L}} N_{e, c^{\prime}, c, t-\theta}^{L} \forall e \in E, s \in S, c \in C, t \in T
\end{aligned}
$$


In the above equations, the parameters $\mu_{e, r, p, \theta}^{P}$ and $v_{e, p, s, c, \theta}^{P}$ represent the amount of resource $r$ (state $s$ of colour $c$ ) of product $p$ produced in equipment $e$ at a time $\theta$ relative to the start of the processing task. Parameters $v_{e, c, s, c^{\prime}, \theta}^{L}$ and $\bar{v}_{e, c, c^{\prime}, s, \theta}^{L}$ are the equivalents of $v_{e, p, s, c, \theta}^{P}$ for the cleaning tasks, while parameter $\tau_{e, p}^{P-1 B}$ represents the duration of the first mixing batch for mixers $(e \leq 3)$ and the total processing time $\left(\tau_{e, p}^{P}\right)$ for extruders. Parameter $\tau_{e, c, c^{\prime}}^{L}$ represents the required cleaning time in equipment $e$ to change from colour $c$ to colour $c^{\prime}$. Finally, to account for equipment usage resulting from orders that began in the previous time horizon, parameter $\gamma_{e, s, c, t}$ is used. The excess variables $R_{r, p, t}$ are positive continuous variables while the excess variables $S_{e, s, c, t}$ as well as the extent variables $N_{e, p, t-\theta}^{P}$ (for processing tasks) and $N_{e, c, c^{\prime}, t-\theta}^{L}$ (for cleaning tasks) are of the binary type. Variables $N B_{e, c, t}$ are also binary and represent the consumption, at time $t$, of the dirty state of colour $c$ in equipment $e$ (if extruder). By using these variables, degenerate solutions near the end of the time horizon are avoided.

Excess resource constraints: In the beginning of the time horizon all raw materials are available in an amount equal to the order size $\left(M_{p}\right)$. At that point, all intermediates and final products are unavailable and the state of all equipments is neutral:

$$
\begin{aligned}
& R_{1, p, 0}=M_{p} \forall p \in P \\
& R_{r, p, 0}=0 \forall r \in R, r>1, p \in P \\
& S_{e, s, c, 0}=0 \forall e \in E, s \in S, c \in C
\end{aligned}
$$

To reduce the number of degenerate solutions, the beginning of the extruding tasks is made to coincide with the end of the corresponding mixing tasks. Also, the cleaning tasks are executed immediately after the end of the processing tasks.

$R_{r, p, t}=0 \forall r \in R, 1<r<5, p \in P, t \in T$

$S_{e, 2, c, t}=0 \forall e \in E, c \in C, t \in T$

Objective function: Two alternative objective functions will be used. The first, production maximisation, will be used to select from the set of total orders, those that can be produced in a given time horizon. Then, the second objective will be used to minimize product delays among schedules with minimum cleaning times.

$$
\begin{aligned}
& \max \sum_{\substack{t \in T \\
t=|T|}} \sum_{p \in P} R_{5, p, t} \\
& \min \sum_{t \in T} \sum_{c \in C} \sum_{\substack { c^{\prime} \in C \\
\begin{subarray}{c}{e \in E \\
e>3{ c ^ { \prime } \in C \\
\begin{subarray} { c } { e \in E \\
e > 3 } }\end{subarray}} N_{e, c, c^{\prime}, t}^{L} \tau_{e, c, c^{\prime}}^{L} T U N I T+\sum_{p \in P} \frac{T R_{p}}{|P|^{3}}
\end{aligned}
$$

The tardiness of each order ( $T R_{p}$, a positive continuous variable) is given by:

$$
T R_{p} \geq \sum_{t \in T} \sum_{\substack{e \in E \\ e>3}} N_{e, p, t}^{P}\left(T_{t}+\tau_{e, p}^{P} T U N I T\right)-d_{p} \forall p \in P
$$

where $d_{p}$ represents the due date of product $p, T_{t}$ the absolute starting time of interval $t$ and TUNIT the length of each time interval. 


\section{A User Friendly Interface}

To handle the scheduling problem a user-friendly software tool was developed. By user-friendly we mean easy data input and generation of the problem's solution without going through its complex modelling aspects. The scheduling tool has two components: i) the general scheduling model (described in section 3.2), implemented in GAMS and ii) an interface to the GAMS software. Microsoft Excel was chosen as the interface since most users are familiarised with spreadsheets and because it has a programming tool associated with it, Visual Basic. Any problem is solved in four steps. In the first step, the problem data is provided in a worksheet of the Excel spreadsheet. In the second step, the data is analysed in order to reduce the number of colour tones to consider and converted to the GAMS format. The problem data is then included in the general GAMS input file, which is solved in the third step. Once the optimal solution is reached, the results are exported to Excel and displayed in the form of Gantt charts.

\section{Model Solving}

A real case study will be used to illustrate the behaviour of the model. It consists of nineteen products and a horizon of one week (the plant operates 5 days a week and 9 hours per day). Product data is provided in Table 1. Due to previous orders, the extruders only become available after 450 (CPE-001), 330 (CPE-002) and 90 minutes (CPE-003) and still require cleaning (from preto, branco and amarelo, respectively). The OSL solver solved the resulting models on a Pentium III-450 MHz machine.

Table 1-Product data (demand in $\mathrm{kg}$, due date in days)

\begin{tabular}{cccccccc}
\hline Product & Colour & Demand & Due Date & Product & Colour & Demand & Due Date \\
\hline 1 & Verde & 500 & 2 & 11 & Vermelho & 500 & 3 \\
2 & Verde & 900 & 3 & 12 & Preto & 500 & 4 \\
3 & Verde & 150 & 4 & 13 & Verde & 3000 & 3 \\
4 & Azul & 250 & 5 & 14 & Vermelho & 400 & 2 \\
5 & Azul & 150 & 2 & 15 & Castanho & 250 & 2 \\
6 & Azul & 450 & 1 & 16 & Castanho & 150 & 5 \\
7 & Azul & 100 & 4 & 17 & Vermelho & 100 & 4 \\
8 & Azul & 150 & 3 & 18 & Castanho & 1000 & 4 \\
9 & Amarelo & 3000 & 5 & 19 & Cinzento & 3000 & 3 \\
10 & Amarelo & 1250 & 1 & Total & & 15800 & \\
\hline
\end{tabular}

Table 2- Computational statistics

\begin{tabular}{ccc}
\hline Objective function & Eq. 8 & Eq. 9 \\
\hline TUNIT (min) & 15 & 5 \\
Time Intervals & 180 & 540 \\
Integer variables & 65639 & 119249 \\
Continuous variables & 99356 & 185900 \\
Constraints & 31317 & 62770 \\
Obj. relaxed MILP & 13462.5 & 258.49 \\
Obj. MILP & 13450 & 264.78 \\
CPU (s) & 7670 & 16732 \\
\hline
\end{tabular}

When solving the problem using the first objective function (equation 8 ) it is found that it is impossible to produce all 19 products. A maximum of $13450 \mathrm{~kg}$ was obtained, corresponding to the sum of the demands of products $1,2,3,9,10,13,14,15,18,19$. These products are then selected for production. Next,

in order to minimise changeover times, the problem is solved using the second objective function (equation 9). A lower discretisation of the time interval is chosen ( 5 instead of 15 minutes) in order to reduce the errors involved in rounding the cleaning and 
processing times to a multiple of the interval length. The optimal schedule is given in Figure 3. As expected, products with the same colour tone are adjacent in the schedule. Product delays are the following: 2.94, 0.43, 1.86, 1.99, 0.92 and 0.71 days for products $1,10,13,15,18$ and 19, respectively. The problem statistics are given in Table 2.

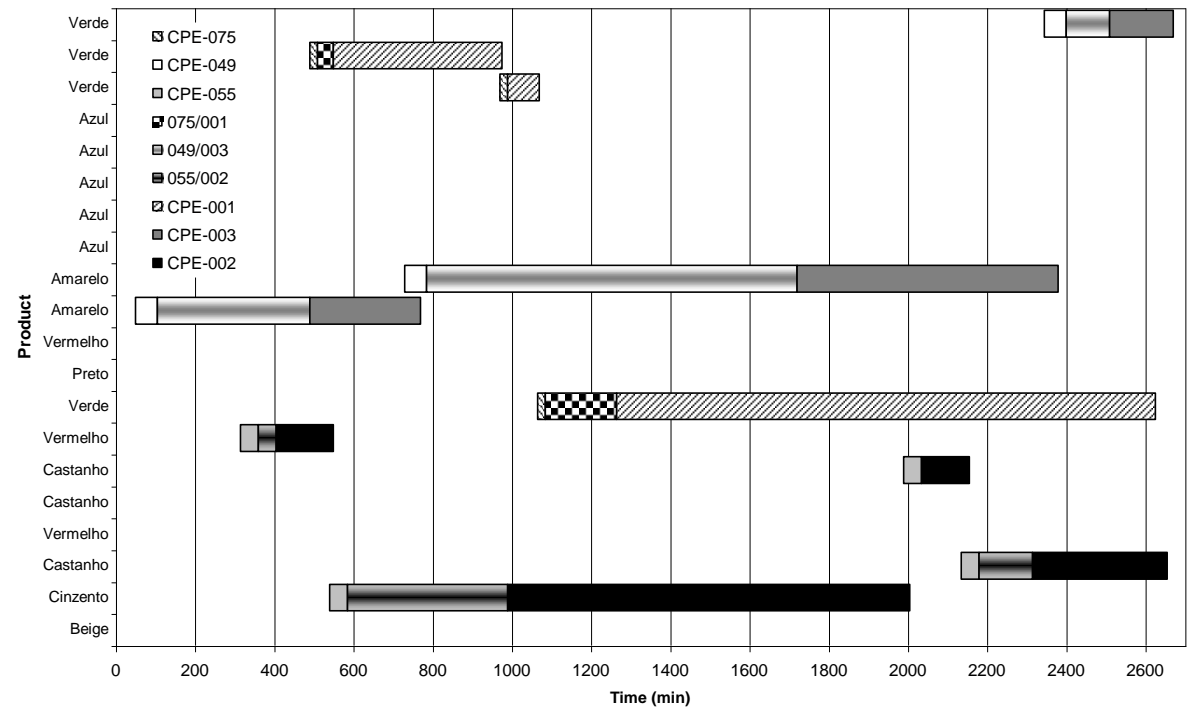

Figure 3- Optimal schedule

\section{Conclusions}

This paper addresses the short-term scheduling problem of a polymer compounding plant. A model, based on a RTN discrete-time formulation was developed to handle the problem. The process superstructure consists of three production lines for each product and all the cleaning possibilities for each equipment item. In order to reduce the number of cleaning possibilities, an algorithm was developed which, by looking at the cleaning times between colours involved in a given set of products, groups, if possible, two or more colours into one new group. The scheduling model was coupled with Microsoft Excel so as to provide a user-friendly interface. Using the production maximisation criterion, the developed software tool selects, from a given set of orders, those most suitable to be produced in a given time horizon. Then, for the selected orders, the optimal schedule that minimises product delays among schedules with minimal changeover times is generated.

\section{References}

Castro, P., 2001, Optimal Short-Term Scheduling of Industrial Batch Processes. PhD

Thesis. Instituto Superior Técnico, Universidade Técnica de Lisboa, Portugal.

Dimitriadis, A., Shah, N. and Pantelides, C., 1998, Comp. Chem. Eng., 22, S563.

Pantelides, C. C., 1994, Unified Frameworks for the Optimal Process Planning and Scheduling. In Proc. $2^{\text {nd }}$ Conference on FOCAPO. CACHE Publications, New York. 